\title{
Study of Listening Training to improve auditory ability for elderly people
}

\author{
Shinichiro Asayama ${ }^{\mathrm{a}^{*}}$, Yuto Tanaka ${ }^{\mathrm{a}}$, Yoshihisa Nakatoh ${ }^{\mathrm{a}}$ \\ ${ }^{a}$ Kyushu Institute of Technology \\ 1-1, Sensui-cho, Tobata-ku, Kitakyushu-shi, Fukuoka, 804-0015, Japan \\ *p349401s@mail.kyutech.jp
}

\begin{abstract}
The elderly are difficult to have a smooth communication, because of their ears and brain declines with aging. In previous research, "technical listening training" has been proposed as a means of auditory-ability improvement. However, whether it is effect way to improve the speech intelligibility of the elderly was not studied yet. In this paper, we proposed a listening training method in order to improve the decline of the brain involved in the hearing of the elderly, and evaluated training effect through comparison with the adults. Listening training consists of five items of discrimination training (pitch, loudness, ITD, ILD, and the length of the sound). The training period is 15 days. Subjects consisted of five males in their 20s, and five males in their 70s. We compared their speech intelligibility before and after training. As a result, in the case of the younger adults, an improvement of about $10 \%$ was observed in SNR -15, $-10 \mathrm{~dB}$, and in the case of the elderly, an improvement of about $20 \%$ was observed in listening level 40, $50 \mathrm{~dB}$.
\end{abstract}

Keywords: auditory ability, auditory training, the elderly, speech intelligibility.

\section{Introduction}

In recent years, the issues affecting Japan have included the declining birthrate and aging society. The elderly have various problems and worries about their life and health. One of them, mishearing similar sounds is a great problem for the elderly. The reasons for mishearing are hearing loss and declining auditory ability. Hearing ability is the ability to recognize sounds by the ear. The hearing ability of the elderly declines by the deterioration of the hair cells in the inner ear. Auditory ability is the ability to understand sounds via the brain. The auditory ability of the elderly decreases with the decline of brain function associated with aging. Hearing aids are recommended to compensate for hearing loss. Hearing ability of the elderly has been improved by use of hearing aids. However, mishearing does not necessarily decrease even if hearing ability has been improved. If sounds are not correctly understood, mishearing occurs. Therefore, compensating for both hearing ability and auditory ability is necessary to decrease mishearing. Now, auditory training has been proposed to improve auditory ability in the previous study. Training in the previous study was used to understand characteristics of sounds for students with the aim at experts of sound ${ }^{(1,2)}$ and the faculty of liberal arts students $^{(3,4)}$. In addition, a support system for hearing aids is applied to get used to sounds from hearing aids ${ }^{(5)}$. This system is used as a part of auditory training. However, the training system is not applied to compensate the declining auditory ability of the elderly.

In this paper, we proposed a training application for smartphones to compensate the declining auditory ability of the elderly. We investigated a compensatory effect of the declining auditory ability of the elderly by proposing training. A speech intelligibility test was used to investigate the effect of our training. In addition, we confirmed the training's effect during training and after training ( 3 months later and 9 months later).

\section{Proposed training methods}

We investigated training methods in the previous study. As a result, we knew roughly two types of training that have been used in the previous study. Two types of training method are the discrimination training and the identification training. The discrimination training is a training to train the ability to notice the subtle difference in sound. Specific example of the discrimination training is to discriminate 
pitch. The discrimination training of pitch is training to select a high-pitched sound from two sounds. The identification training is the training to train the ability to recognize acoustic characteristics. Specific example of the identification training is to identification of pitch. The identification training of pitch is training to distinguish the numerical value of frequency from a sound. We thought that to discriminate the subtle difference of sound is necessary for discrimination the characteristic of voice. Therefore, we decided to use discrimination training for our proposed training. In addition, training of a previous study is very difficult and takes a long time because of exclusive training. Therefore, training of previous study is a big burden on the elderly and difficult to continue training. Therefore, we chose training items from training of previous study. Chosen training items are most effective training items for compensating auditory ability of the elderly. We finally decided to use 5 items of the discriminate training. Table. 1 is shown training items to use at our proposed training. In training items, evaluation of the length of the sound and ITD (Interaural Time Difference), ILD (Interaural Level Difference) were selected with reference to the software mentioned ${ }^{(5)}$. High-tone sounds about $8,000 \mathrm{~Hz}$ are not heard very well for one of the elderly. Therefore, we used one-second pure tone $(1,000$ $\mathrm{Hz}$ ) at the training. The individual difference of the elderly is large in the hearing ability at ears and auditory ability at brain. Therefore, the hearing method of the training sound is individual hearing using headphones.

\section{Implementation of proposed training}

Subjects used for comparison were five males from 72 years old to 77 years old, and five normal-hearing males from 21 years old to 22 years old. Training period was defined as 15 days, schedule of training was executed so as to not make intervals of the training days. Training for the young adults had 10 questions each, five items being training. In the case of the elderly people, training of pitch and loudness and length of the sound were each 10 questions and training of ITD and ILD were 10 questions

Table 1. Training items

\begin{tabular}{|c|c|}
\hline \multirow{4}{*}{$\begin{array}{c}\text { Training } \\
\text { items }\end{array}$} & Poudness \\
\cline { 2 - 2 } & Length of sound \\
\cline { 2 - 2 } & Interaural time difference (left and right) \\
\cline { 2 - 2 } & Interaural time difference (left and right) \\
\hline
\end{tabular}

for both the left and right ear, therefore the elderly people had a difference in the hearing level of the left and right as compared to the young adults. We defined six stage training difficulty levels depending on displacement of training sound. In the case of the young adults, there was not much individual difference in training difficulty level, therefore we presented the same training difficulty level questions to all subjects and evaluated the training results by the change in the percentage of correct answers for 15 days. In the case of the elderly people, there was great individual difference in training difficulty level. Therefore, we changed the training difficulty level to the correct answer rate of the training, evaluating the training results by the change of training difficulty level. In addition, the first-time and second-time trainings are the period to get used to the training, and it was decided not to be used for evaluation of improvement in auditory ability.

\section{The effect of improving auditory ability}

\subsection{The effect of improving the speech intelligibility by training}

In this section, we evaluated improving auditory ability by proposed training. We used speech intelligibility at valuation. Speech intelligibility tests were performed four times in total (before training, five days, 10 days and 15 days). We compared with each test results. Inspection speech used the 67-S word table. The 67-S word table is used a hearing test of Japanese syllable. One set of hearing test is twenty Japanese syllables. In the case of the young adults, hearing ability at ear and auditory ability at brain do not decrease as compared to the elderly people. Therefore, we set four stages of SNR $(-15,-10,-5$, and $0 \mathrm{~dB})$ at the ear by giving a white noise. We carry out hearing test by stages of SNR. In the case of the elderly people, hearing ability at ear and auditory ability at brain do not decrease as compared to the young adults. Therefore, we set four stages $(40,50,60$, and $70 \mathrm{~dB})$ of listening sound pressure of the target sound. We carry out hearing test by stages of listening sound pressure. The test results are shown of the young adults in Fig. 4. The test results are shown of the elderly people in Fig. 5. The vertical axis in each figure is the percentage of correct answers, and the horizontal axis is training days. In Fig. 4, the young adults did not observe large increases for five days, however, the young adults observed an improvement of about $10 \%$ in SNR $-15,-10 \mathrm{~dB}$ at 10 days and 15 days. Improvement of speech 
intelligibility on small SNR increases larger than large one. In Fig. 2, an improvement of about $10 \%$ was observed in the elderly people on the listening sound pressure 40, $50 \mathrm{~dB}$ at five days, and an improvement about $20 \%$ on the listening sound pressure $40,50 \mathrm{~dB}$ at 15 days. Improvement of speech intelligibility on small listening sound pressure increases larger than large one. The speech intelligibility of improvement rate in elderly people is remarkably larger than the young adults. Elderly people decrease auditory ability as compared to young adults. Therefore, we considered that the improvement of auditory ability in elderly people by training appears to be larger than young adults'.

\subsection{The effect in speech intelligibility by practice to test}

In the previous section, an effect of improved speech intelligibility by training was confirmed. In this section, we evaluated the effect of improvement speech intelligibility by practice to hearing test. This evaluation is for proof of useful in previous section. Therefore, we performed a speech intelligibility test without training. The interval of test was same at training (before training, five days, 10 days and 15 days). Subjects were five males from 70 years old to

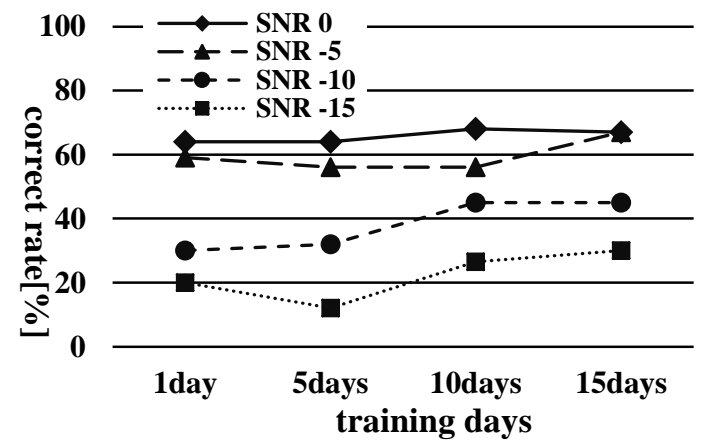

Fig. 1. Speech intelligibility by training (young adults)

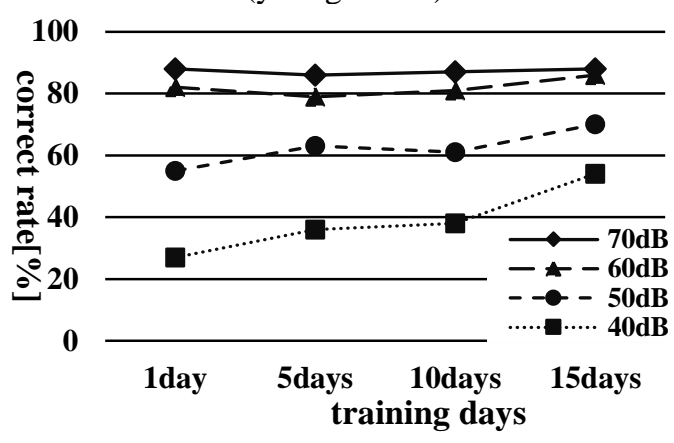

Fig. 2. Speech intelligibility by training (the elderly)
78 years old and five normal-hearing males from 22 years old to 24 years old. The test results of the young adults are shown in Fig. 3, and the test results of the elderly people are shown in Fig. 4. The vertical axis in each figure is the percentage of correct answers and the horizontal axis is training days. Improvement in the percentage of correct answers is not observed in Fig. 3 and Fig. 4. As a result, improvement in speech intelligibility test is by the training.

\subsection{The effect in speech intelligibility after the training}

When time more than three months passed after the training end, we investigated whether the training effect at speech intelligibility was sustained. Subjects included five males in their 20sand four males in their 70s who were trained in previous evaluation. Speech intelligibility tests after the training end were performed after three months and nine months. The effect of the speech intelligibility at the young adults after the training end is shown in Fig. 5. The effect of the speech intelligibility at the elderly people after the training end is shown in Fig. 6. In Fig. 5, improvement of speech intelligibility before and after training was observed at SNR -10howeverthe effect of speech intelligibility after the training end was not observed.

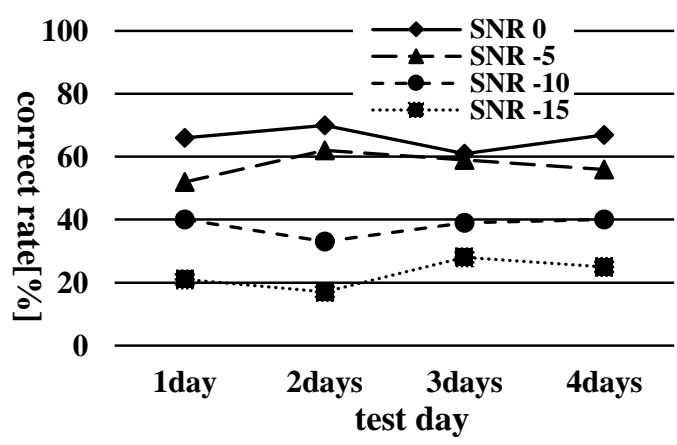

Fig. 3. Speech intelligibility without training (young adults)

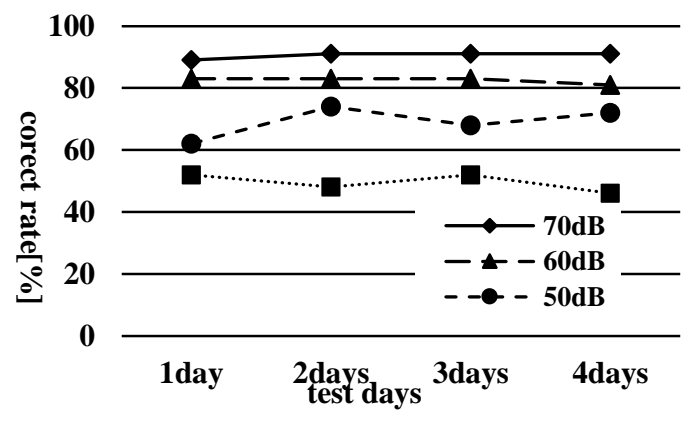

Fig. 4. Speech intelligibility without training (the elderly) 
The increase in speech intelligibility before and after training at SNR -5, 0 were not observed, also, decrease of speech intelligibility after the training end at SNR -5, 0 were not observed. In Fig. 6, a large increase in training effect before and after training at $40 \mathrm{~dB}$ and $50 \mathrm{~dB}$ were observed, however, a large decrease in training effect was observed after the training end. In Fig. 6, increase in speech intelligibility before and after training at $60,70 \mathrm{~dB}$ were not observed, also, decrease of speech intelligibility after the training end at 60, $70 \mathrm{~dB}$ were not observed. In Fig. 5 and Fig. 6, the results of the elderly people were similar to that of the young adults. Thus, the large increase speech intelligibility both the young adults and the elderly people does not persist without auditory training. In addition, the degree of improvement in the elderly people was greater than the young adults, as well as the degree of decrease training effect in the elderly people. Therefore, the auditory ability of elderly people decreases as compared to the auditory ability of young adults.

\section{Conclusions}

In this paper, we propose an auditory training method for the purpose of improving the auditory ability of elderly people, and have investigated the effect on elderly people and young adults. In addition, we have confirmed what kind of influence on training has when spaced from training. As a result, improvement of speech intelligibility by training in young adults and elderly people was observed. In the future, evaluation of training effect is expected for each training part, and to implement a training application, evaluate
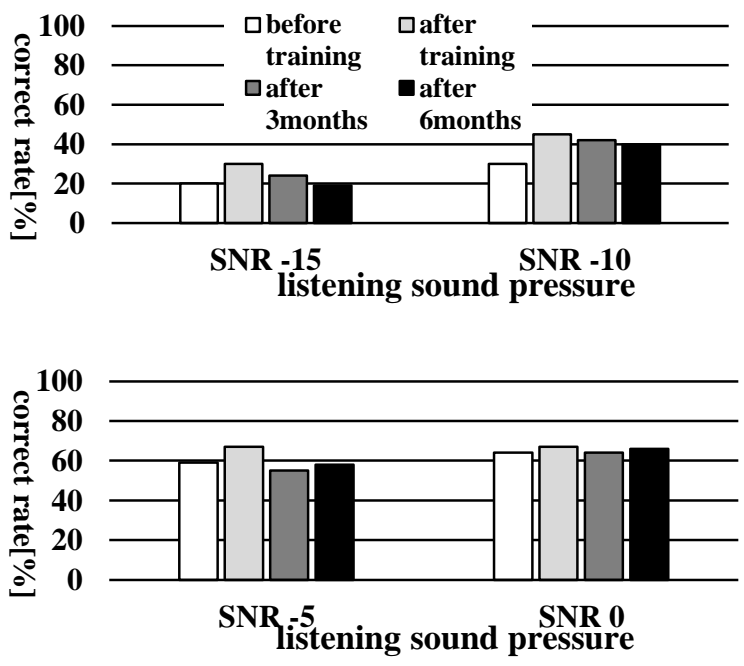

Fig. 5. Speech intelligibility after training (younger adults) training effect by using the application.

\section{Acknowledgment}

Part of this study was conducted with the assistance of the Ministry of Education Science and Research Grants Fund grant (NO.26350661).

\section{References}

(1) Shin-ichiro Iwamiya : "Technical listening training : Training to improve sensitivity to sounds" The journal of the acoustical society of Japan, vol. 69, no. 4, pp. 197-203, 2013

(2) Shin-ichiro Iwamiya, Yoshitaka Nakajima, Kazuo Ueda Kazuhiro Kawahara and Masayuki Takada : "Technical listening training: Improvement of sound sensitivity for acoustic engineers and dound desighners" Acoust. Sci. \&Tech., 24, pp. 27-31, 2003

(3) Akira Nishimura : "Effects of ear training on lectures of audio information processing for nontechnical undergraduates" The journal of the acoustical society of Japan, vol. 62, no. 3, pp. 208-213, 2006

(4) Akira Nishimura : "Auditory training system that uses TCP/IP networks and WWW browsers" The journal of the acoustical society of Japan, vol. 70, no. 5, pp. 252-259, 2014

(5) Arthur Boothroyd : "Adapting to changed hearing: the potential role of formal training" Jounal of the American Academy of Audiology, vol. 21, No. 9, pp.601-611, 2010
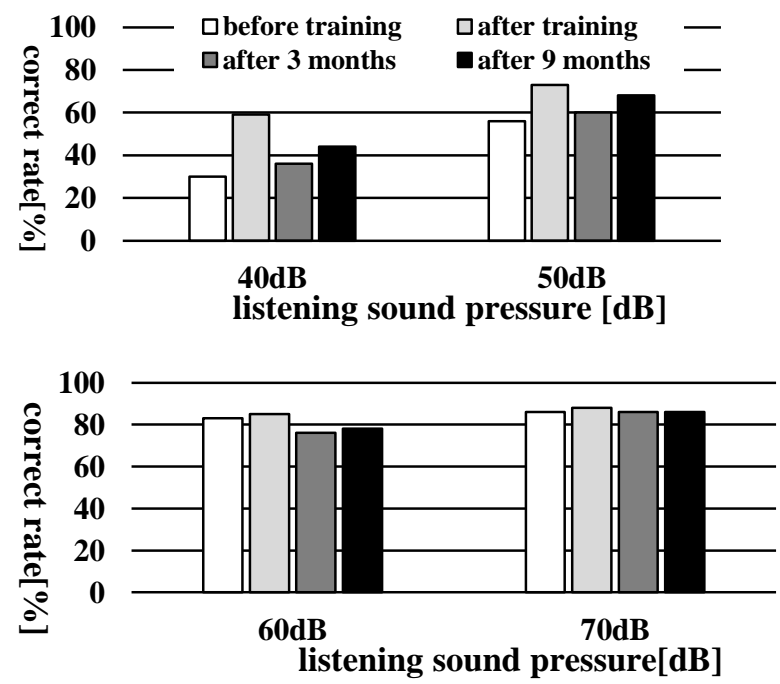

Fig. 6. Speech intelligibility after training (the elderly) 